\title{
Reliability of Measuring Anomalous Experience: The Bonn Scale for the Assessment of Basic Symptoms
}

\author{
Anne Vollmer-Larsen ${ }^{\mathrm{a}, \mathrm{b}} \quad$ Peter Handest $^{\mathrm{b}} \quad$ Josef Parnas $^{\mathrm{a}, \mathrm{b}}$ \\ ${ }^{a}$ Danish National Research Foundation, Centre for Subjectivity Research, University of Copenhagen, Copenhagen, \\ and ${ }^{b}$ Department of Psychiatry, Hvidovre Hospital, Brøndby, Denmark
}

\section{Key Words}

Subjective experiences · Schizophrenia prodrome $\cdot$ Bonn

Scale for the Assessment of Basic Symptoms, reliability

\begin{abstract}
Background: Studying subjective experience, apart from preformed self-rating questionnaires, has nearly vanished in psychiatry, partly due to reliability concerns. Recent research in early detection of schizophrenia has entailed an increasing interest in the subtle experiential anomalies that may assist in identifying the patients at risk of psychosis. Some of these anomalies are described in the Bonn Scale for the Assessment of Basic Symptoms (BSABS). We examined the reliability of this instrument. Sampling and Method: 18 hospitalised patients accepted to participate in a psychopathological interview assessing BSABS items, affective and psychotic symptoms. Results: Out of the total 79 BSABS items examined, we found an interrater reliability kappa $>0.60$ in 68 items (86\%). Conclusion: Good reliability can be achieved using BSABS.

Copyright $\odot 2007$ S. Karger AG, Base
\end{abstract}

\section{Introduction}

Studying patients' subjectivity has become limited in scope and methodology in the science of psychopathology because of unilateral concerns about reliability issues
[1]. However, certain alterations of subjective experience have been proposed to 'be listed along with signs such as Bleuler's associative loosening' [2]. The interest in the prodromal detection of schizophrenia has entailed increasing understanding of the importance of subtle, nonpsychotic anomalies of experience as symptoms that may accurately identify individuals at risk of future psychosis [3]. By the same token, the lack of standard psychopathological resources to address such subjective experience is becoming strikingly visible $[4,5]$.

One important exception is the work of Gross et al. [6], who have described and defined a wide range of these symptoms in the Bonn Scale for the Assessment of Basic Symptoms (BSABS). They coined them as 'basic symptoms' on the assumption that these features were pre-psychotic antecedents of full-blown psychosis and hence proximate to the underlying hypothetical causal biological dysfunction [7]. The relevance of basic symptoms for the prediction of schizophrenic psychosis has been investigated by Klosterkötter et al. [8] and some basic symptoms have been included in the early detection and intervention research.

Here we present data on the interrater reliability of the Danish version of the BSABS as a lifetime examination.

\section{Materials and Methods}

Eighteen patients hospitalised in an open ward were included. Inclusion criteria were ability to participate in an approximately

\section{KARGER}

Fax +41613061234 E-Mail karger@karger.ch www.karger.com

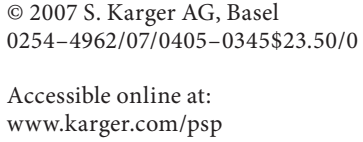

Anne Vollmer-Larsen

Department of Psychiatry, Hvidovre Hospital

Brøndbyøstervej 160

DK-2605 Brøndby (Denmark)

Tel. +45 3632 3817, Fax +45 3632 3974, E-Mail Anne.Vollmer-Larsen@hh.hosp.dk 
Table 1. Reliability of the BSABS

Dynamic deficiencies: A

A.2 increased need for sleep

A.6.1 diminished sense of pleasure

A.6.2 changes in experience of own emotions

A.6.4 diminished need for contact

A.7.1 diminished ability for contact

A.7.2 changes in ability for self-expression

A.8.1 decreased threshold for changes

A 8.2 decreased threshold

A.8.3 decreased threshold for stress

A.8.4 loss of ability for shared attention

Dynamic deficiencies: B

B.2 increased emotional reactivity in response to $1.00^{* * *}$ routine daily events

B.2.2 increased emotional reactivity in response to behaviour of others concerning the patient

B.2.3 increased emotional reactivity in response to 'the suffering of other persons'

B.3.2 obsessive phenomena

B.3.3 phobia

B.3.4 psychic depersonalisation

Anomalies of cognitive, perceptual and motor experience: $\mathrm{C}$

Anomalies of cognitive experience

C.1.2.1 perseveration of thoughts

C.1.2.2 perseveration of thoughts - fantasy

C.1.3 thought pressure

C.1.4 thought blockages

C.1.5 disturbed concentration

C.1.6.1 disturbance of receptive language - visual

C.1.6.2 disturbance of receptive language - acoustic

C.1.7 disturbance of expresssive language function

C.1.8 disturbance of ultra-short-term memory

C.1.9 disturbance of short-term memory

C.1.10 special disturbances in long-term memory

C.1.11 memory disturbances - not classified

C.1.12 slow and difficult thinking

C.1.13 disturbance of thought initiative or thought intentionality

C.1.15 changes in discrimination between ideas and perception

C.1.16 disturbance in understanding of symbols

C.1.17 unstable ideas of reference

Anomalies of perceptual experience - visual

C.2.1.1 unclear sight - phasic

C.2.1.2 momentary blindness

C.2.1.3 partial vision

C.2.2.1 sensitivity to light

C.2.2.2 photopsia

C.2.3.1 nearsight

C.2.3.2 changes in size

$0.45^{* * *}$

$0.45^{* * *}$

$0.67^{* * *}$

$1.00^{* * *}$

$0.92^{* * *}$

$1.00^{* * *}$

$0.73^{* * *}$

$0.74^{* * *}$

$0.60^{* * *}$

$0.80^{* * *}$

$0.89^{* * *}$

$0.68^{* * *}$

$0.71^{* * *}$

$0.89^{* * *}$

$0.83^{* * *}$

$0.87^{* * *}$

$1.00^{* * *}$

$0.74^{* * *}$

$0.69^{* * *}$

$0.87^{* * *}$
C.2.3.3 changes in form

C.2.3.4 changes in colour

C.2.3.5 changes in others' face or body

C.2.3.6 mirror-related phenomena

C.2.3.7 movements of objects experienced as related to own movements

C.2.3.8 diplopia, oblique vision $0.21^{* * *}$

C.2.3.9 disturbances in estimation of distances or size $0.43^{* * *}$

C.2.3.10 disintegration in perception of linearity of $0.61^{* * *}$ contours

C.2.3.12 abnormally long-lasting retinal after-image $\quad 0.75^{* * *}$

Anomalies of perceptual experience - auditory, olfactory,

gustatory or tactile

C.2.4.1 sensitivity to sound

C.2.4.2 acoasma

C.2.5.1 changes in auditory intensity or quality

C.2.5.2 abnormal acoustic irritation

C.2.6.1 olfactory changes

C.2.6.2 gustatory changes

C.2.6.3 tactile changes

C.2.7 disturbance in grasping the significance of observed objects

C.2.8 heightened perception

$0.72^{* * *}$

$0.62^{* * *}$

$0.82^{* * *}$

$0.44^{*}$

$0.89^{* * *}$

$1.00^{* * *}$

$0.88^{* * *}$

$0.78^{* * *}$

$1.00^{* * *}$

\section{Anomalies of motor experience}

C.2.9 captivation of attention by visual details $1.00^{* * *}$

C.2.10 disturbance of awareness of continuity of own $1.00^{* * *}$ actions

C.2.11.1 derealisation

$0.76^{* * *}$

C.3.1 automatism

C.3.2 motor blocking

D. 4

$1.00^{* * *} \quad$ D. 5

D.6

94\% D.7 sensations of movement

disautomatisation

Coenaesthesias

D.1 unusual bodily sensations of numbness and

$0.54^{* * *}$ stiffness

D.1 somatic depersonalisation

experience of motor paresis

unusual sensations of bodily pain

migrating sensations

electric sensations

thermal sensations

sensations of abnormal heaviness, lightness

$0.75^{* * *}$

$0.78^{* * *}$
$0.80^{* * *}$

$1.00^{* * *} \quad$ D. 8

or emptiness, of falling or sinking, levitation

or elevation

0.73*** D.9 sensation of extension, diminution, shrinking, $0.80^{* * *}$

enlargement or constriction

$0.87^{* * *} \quad$ D.10 kinesthetic sensations $0.89^{* * *}$

$0.74^{* * *} \quad$ D.11 vestibular sensations $0.87^{* * *}$

$0.79^{* * *} \quad$ D.12 dysaesthesias provoked by sensory or tactile $0.57^{* * *}$

$0.64^{* * *} \quad$ stimulations

$0.65^{* * *} \quad$ D.14 dysaesthetic crisis

$0.61^{* * *}$

Figures indicate kappa values. ${ }^{\star} \mathrm{p}<0.05 ;{ }^{* \star} \mathrm{p}<0.01 ;{ }^{* *} \mathrm{p}<0.001$. 
3-hour psychopathological interview, no primary abuse diagnosis, no mental retardation nor organic aetiology of the psychiatric illness. Both raters (A.V.L., P.H.) were specialists in psychiatry with special interest in psychopathology. One of the raters had been formally trained for the use of the BSABS under the supervision of one of the developers of the scale (Klosterkötter). Prior to the interviews, 12 training sessions were completed. Items were individually documented during the interview and raters alternated between being interviewer and observer.

The BSABS is a semi-structured interview consisting of 92 principal items described in a prototypical manner, supplemented by differential-diagnostic guidelines, examples of questions and suggestions of probes. Symptoms are divided into stage 1 and 2 basic symptoms. The stage 2 basic symptoms are found to be more specific to schizophrenia spectrum disorders than to other psychiatric disorders [6]. For the study, we selected 59 basic symptoms (78 items because of specific subcategories) including all stage 2 basic symptoms covering disturbances of thinking, perception, motor experiences and coenaesthesias (abnormal bodily experiences). Each item is usually scored as absent, doubtfully present or definitely present; due to a clinical setting with restricted time, we chose to include a scoring of unknown/not sufficiently investigated. A full BSABS interview takes up to $180 \mathrm{~min}[6,9]$. Lifetime was chosen as the time span of symptoms, but it may be varied according to the study purpose. The BSABS was embedded a more comprehensive clinical interview covering sociodemographic information, life history, psychotic, 'neurotic' and affective symptoms - essentially based on the skeleton of the OPCRIT and the SADS-L $[10,11]$ and completed by the PANSS scorings and scorings of expressive features, thus forming a composite, coined for daily use as the Hvidovre Checklist, and employed as a standard in our studies. The interviews are always performed in a semi-structured way, i.e. in a systematic but context-adequate way, conductive to maximally detailed self-descriptions rather than to binary yes/no answers.

The reliability was assessed by calculating Cohen's kappa values, which measure agreement between raters corrected for chance agreement and taking frequency into account. In the absence of variation in the ratings, e.g. in rating an important, but a rare symptom, kappa values can sometimes not be calculated and per cent agreement has to be used $[12,13]$.

The patients were 11 males (age $20-45$ years, mean 30.4, median 29) and 7 females (age 22-34 years, mean 29.6, median 32) diagnosed with schizophrenia (8 patients), schizotypal disorder (3 patients), personality disorder (4 patients) and affective disorder (3 patients). Duration of illness was 1-209 months (mean 39.8, median 26). The level of education was as follows: 5 patients 9 years of school, 1 patient 10 years of school, 6 patients 12 years of school, 4 patients studied at a university and 1 had graduated with a university degree. Five patients received antipsychotic drugs, 3 antidepressants and 3 a combination of both.

\section{Results}

The reliability of the entire interview was quite good, as measured by the interclass correlation coefficients (ICCs) for the PANSS positive, negative and total symp- tom scales (ICC for PANSS positive symptoms 0.99, ICC for PANSS negative symptoms 0.95 and ICC for PANSS total symptoms 0.97 ).

Reliability of the BSABS is itemised in table 1. Out of 79 items, we found a fair kappa value $(0.21-0.40)$ in 2 (a form of increased emotional reactivity and a rare visual perception disturbance). A discrepancy in these particular ratings was caused by the fact that one rater considered the symptom as doubtfully present while the corater considered it as absent. In the totality of $78 \times 18$ ratings, there were only 12 clear-cut disagreements, with one rater scoring an item as absent and the other as definitely present.

A moderate kappa value (0.41-0.60) was found in 8 items, a good kappa value (0.61-0.80) in 34 items, and a very good value (0.81-1.00) in 34 items. For 1 item, kappa could not be calculated and was expressed as 94\% agreement [13].

\section{Discussion}

The current focus on the prodromal detection of schizophrenia has highlighted the need for a useful approach to describing alterations of subjective experience. The BSABS has been, until recently, the only such instrument - if we disregard the self-rating approaches. In this investigation, the reliability of BSABS items was investigated, when the BSABS is integrated into a more comprehensive diagnostic assessment. We found high interrater kappa values for nearly all items. The study did not address the issues of test-retest reliability.

The observed level of inter-rater reliability demonstrates that it is possible to incorporate an assessment of anomalous subjective experience into a more general clinical-diagnostic interview performed within a restricted time frame by trained psychiatrists. From these findings, it follows that reliability concerns should not prevent scientific investigation of altered subjectivity or consciousness. Our results are partly relevant to the recently published interview scheme - the EASE (Examination of Anomalous Self-Experience) - targeting anomalies of self-experience [14] because there are important EASE-BSABS overlaps; the EASE may be considered as a phenomenological extension of the BSABS in the domain of self-experience.

Psychopathology 2007;40:345-348 


\section{References}

1 Parnas J, Bovet P: Research in psychopathology: epistemological issues. Compr Psychiatry 1995;36:167-181.

$\checkmark 2$ Meehl P: Primary and secondary hypohedonia. J Abnorm Psychol 2001;110:188-193.

-3 McGlashan TH, Johannessen JO: Early detection and intervention with schizophrenia: rationale. Schizophr Bull 1996;22:201222.

4 Larsen TK, Friis S, Haahr U, Joa I, Johannessen JO, Melle I, et al: Early detection and intervention in first-episode schizophrenia: a critical review. Acta Psychiatr Scand 2001; 103:323-334.

5 Parnas J: Clinical detection of schizophrenia-prone individuals: critical appraisal. Br J Psychiatry 2005;187(suppl 48):8-18.

6 Gross G, Huber G, Klosterkötter J, Linz M: Bonner Skala für die Beurteilung von Basissymptomen. Berlin, Springer, 1987, p 1995.
7 Klosterkötter J: Basissymptome und End phänomene der Schizophrenie: Eine empirische Untersuchung der psychopathologischen Übergangsreihen zwischen defizitären und produktiven Schizophreniesymptomen. Berlin, Springer, 1988.

8 Klosterkötter J, Hellmich M, Steinmayer EM, Schultze-Lutter F: Diagnosing schizophrenia in the initial prodromal phase. Arch Gen Psychiatry 2001;58:158-164.

9 Gross G, Stassen HH, Huber G, Klosterkötter J: Reliability of the psychopathological documentation scheme BSABS; in Stefanis CN, Rabavilas AD, Soldatos CR (eds): A World Perspective. Amsterdam, Elsevier, 1990, pp 199-203.
10 Endicot J, Spitzer RL: A diagnostic interview: the schedule for affective disorders and schizophrenia. Arch Gen Psychiatry 1978; 35:837-844.

11 Williams J, Farmer AE, Ackenheil M, Kaufmann CA, McGuffin P; the OPCRIT Reliability Research Group: A multicentre inter-rater reliability study using the OPCRIT computerized diagnostic system. Psychol Med 1996;26:775-783.

12 Altman DG: Practical Statistics for Medical Research. London, Chapman \& Hall/CRC, 1991.

13 Bartko JJ, Carpenter WT: On the methods and theory of reliability. J Nerv Ment Dis 1976;163:307-317.

14 Parnas J, Møller P, Kircher T, Jansson L, Handest P, Zahavi D: EASE: Examination of Anomalous Self-Experience. Psychopathology 2005;38:236-258. 